\section{Methods and results}

Necropsy was carried out on 25 patients ( 24 men, 1 woman) who died with AIDS in this hospital. The average age at death was 39.8 years (range 25-60), and none had any skeletal disease or joint symptoms. During necropsy three pieces of synovial tissue were removed from within the right knee cavity and examined using standard histological techniques, including those for elastic, bacteria, fungi, and acidalcohol fast bacilli. Similar control tissue was removed at necropsy from 12 other patients ( 7 men, 5 women) aged 20-88 years (mean 55.9) and processed in the same way. These had died of other causes, including suicide in an otherwise healthy person, and four were known to have osteoarthritis in the right knee.

Each fragment of synovium from the patients with AIDS showed abnormalities. Nineteen showed focal thinning and sometimes loss of the lining synovial surface; immediately underneath and running parallel was a band of collagen, either continuous or interrupted, but occupying at least half of the synovial surface area (see figure $a$ ). In continuity were thick bands of collagen traversing the deep lying adipoconnective tissue. Within the tissue were small arteries which showed endothelial swelling, fibromuscular proliferation of the intima, fragmentation or reduplication of the internal elastic lamina (or both), and proliferation of the elastic fibres in the adventitia (figure $b$ ). The fibromuscular change in the intima was often symmetrical with almost complete obliteration of the lumen, but was sometimes asymmetrical, producing a cushion of intima bulging into the lumen (figure $c$ ). Three further cases showed extensive loss of cells from the synovial surface, while the entire synovium was almost replaced by dense, poorly cellular collagen (figure $d$ ); the arteries were as before. The remaining three patients had a normal surface with only a mild degree of fibrosis in the synovium; the arterial findings were as before. There was no other abnormality in any case and searches for organisms and neoplasia produced negative results. In all the joints the cartilage surface was normal. The synovial tissue from the controls was normal except in those with osteoarthritis, who showed a mild degree of noninflammatory fibrosis.

\section{Comment}

The abnormalities found in these patients-namely, fibrosis attributed to ischaemia from the specific changes in the small arteries-seem to be unique to the synovium in AIDS.

The structure of normal synovial tissue is well described ${ }^{2}$ and its importance in the processes of joint disease is clear. ${ }^{24}$ Brewerton emphasises that a joint, regarded as part of the reticuloendothelial system, is particularly vulnerable to invasion by foreign substances and states that the vascular endothelium is the critical barrier between the rest of the body milieu and the joints. ${ }^{5}$ In joint disease, although a single disease can sometimes be induced by one agent, several different agents are probably more often implicated. In these patients with AIDS the initial insult to the synovium, probably HIV inspired, is likely to be modified by other factors such as the degree of immune suppression.

These joint changes are serious for all patients with AIDS. The histological findings are those of a greatly accelerated aging process in a predominantly young population developing over a short period. Premature aging is an external hallmark of established AIDS and, together with all the other known features, we believe that there is a greatly increased likelihood of potentially disabling degenerative joint disease.

We thank the clinicians of both St Stephen's and the Middlesex Hospitals who have allowed us to study their patients; Mr A J Hall, Mr P Mooney, and Mr D Webber for invaluable technical help; $\mathrm{Mr} \mathrm{M}$ Nelson for photomicrography; and Mrs K Burnett for help with researches and the preparation of the manuscript.

1 Rowe IF, Forster SM, Seifert MH, et al. Rheumatological lesions in one hundred and twenty three individuals with human immunodeficiency virus infection. Qf Med 1989;73:1167-84.

2 Edwards JCW. Synovial structure and function. London: Arthritis and Rheumatism Council, 1988. (Reports on Rheumatic Diseases series 2, No 8.) Gardner DL. The pathology of rheumatoid arthritis. London: Edward Arnold, 1972.

4 Fell HB, Jubb RW. The effect of synovial tissue on the breakdown of articular cartilage in organ culture. Arthritis Rheum 1977;20:1359-71.

5 Brewerton DA. Causes of arthritis. Lancet 1988;ii:1063-6.

(Accepted 22 February 1990)

\section{Hospital for Tropical Diseases, London NW1 OPE \\ Adam S Malin, MRCP,} registrar

\section{Homerton Hospital, London \\ Peter L Cass, MRCOG, senior registrar \\ C N Hudson, FRCOG, consultant}

Correspondence to: Dr Malin.

BrMed F 1990;300:1240-1

\section{Exchange transfusion for severe falciparum malaria in pregnancy}

\section{Adam S Malin, Peter L Cass, C N Hudson}

Falciparum malaria in pregnancy is associated with fetal and maternal death. Intravenous quinine is the main treatment but may reduce the parasitaemia slowly. Exchange transfusion has been used successfully to treat severe and complicated cases of falciparum malaria in non-pregnant patients. ${ }^{1}$ We report on a pregnant woman with severe falciparum malaria who was treated with exchange transfusion.

\section{Case report}

A 21 year old woman of Nigerian origin presented after 26 weeks' amenorrhoea in her first pregnancy with an eight week history of non-specific abdominal pain, which had worsened over the previous 48 hours; her pregnancy had not been booked. She stated later that although most of her earlier life had been spent in Nigeria she had not left England for the previous three years.
On presentation she was delirious and unable to give a history. Her temperature was $39^{\circ} \mathrm{C}$. Examination confirmed signs of pregnancy, but findings were otherwise unremarkable. An abdominal ultrasound scan showed a single viable fetus equivalent to a 33 week gestation. Haematological investigations showed a haemoglobin concentration of $109 \mathrm{~g} / \mathrm{l}$, leucocyte count $9 \times 10^{9} / 1$, and platelet count $93 \times 10^{9} / 1$. Results of biochemical investigations were normal except for a raised bilirubin concentration of $82 \mu \mathrm{mol} / \mathrm{l}$. Clotting studies, a sickling test, and haemoglobin electrophoresis yielded normal results. Examination of urine showed large amounts $(3+)$ of blood, protein, and bilirubin. A blood film showed Plasmodium falciparum: one fifth of the erythrocytes contained parasites.

In the intensive care unit $600 \mathrm{mg}$ of quinine dihydrochloride was given as a four hour intravenous infusion every 12 hours. Four hours after the first infusion she had an exchange transfusion of seven units of blood and a transfusion of four units of fresh frozen plasma over five hours. She remained haemodynamically stable throughout the procedure, and there was no evidence of fetal distress. Moderate uterine activity resolved spontaneously.

Twelve hours after admission the patient was alert, her temperature was $37 \cdot 2^{\circ} \mathrm{C}$, and a blood film showed a 
dramatic fall in the parasitaemia to $0.5 \%$. Forty eight hours after admission her temperature was normal and no parasites were detectable in further films. The quinine was then continued orally for 10 days at a dose of $600 \mathrm{mg}$ every 12 hours.

Her subsequent antenatal course was uncomplicated. Films remained negative for malarial parasites. Four weeks after her admission she was readmitted in spontaneous labour that resulted in a vaginal delivery of a healthy girl weighing $2680 \mathrm{~g}$. The puerperium was uneventful.

\section{Comment}

We believe this to be the first report of successful exchange transfusion for severe infection with $P$ falciparum in pregnancy. Mothers in the second trimester of their first pregnancy are particularly at risk of the complications of malaria. ${ }^{23}$ Exchange transfusion is thought to act by reducing the number of red cells infected with the parasites and may also remove deleterious humoral factors such as tumour necrosis factor, which has been implicated in the pathogenesis of organ failure. ${ }^{+5}$
$P$ falciparum does not have an exoerythrocytic liver phase, and thus clinical malaria that occurs more than a year after the patient has lived in a malarious zone is rare. This patient did not live close to an airport and there was no reason to disbelieve her history. Perhaps the most likely explanation is that a chronic, asymptomatic parasitaemia developed into fulminating disease as a result of the immunosuppression associated with pregnancy.

We thank Dr A D M Bryceson for his help with the manuscript.

1 Hall AP. Exchange transfusion and quinine levels in falciparum malaria Acta Leiden 1987;55:121-8

2 Paluku KM, Moore M, Roy J, Ma-Disu M. Malaria infection in pregnan women in Zaire: the effects and the potential intervention. Ann Trop Med Parasitol 1988;82:113-20.

3 Naing T, Win H, Nwe YY. Falciparum malaria and pregnancy: relationship and treatment response. Southeast Asian f Trop Med Public Health 1988;19: 253-8.

4 Grau GE, Taylor TE, Molyneux ME, et al. Tumour necrosis factor and disease severity in children with falciparum malaria. $N$ Engl f Med 1989;320: $1586-91$.

5 Clark IA, Chaudhri G. Tumour necrosis factor in malaria-induced abortion Am f Trop Med Hyg 1988;39:246-9.

(Accepted 8 February 1990)

\title{
Five year outcome in patients withdrawn from long term treatment with diazepam
}

\author{
Anthony Holton, Peter Tyrer
}

\section{Long Grove Hospital, Horton Lane, Epsom, Surrey \\ Anthony Holton, MRCPSYCH, consultant psychiatrist}

St Charles's Hospital, London W10

Peter Tyrer, FRCPSYCH, senior lecturer

Correspondence to: Dr Tyrer.

Br.Med f 1990;300:12+1-2
Only in the past 10 years has dependence on benzodiazepines in therapeutic doses been recognised as a major problem, ${ }^{\prime 2}$ and information on long term outcome is limited. Ashton found that $92 \%$ of patients had not resumed benzodiazepine treatment 10 months to $31 / 2$ years after stopping long term treatment, but her population was a specialised group referred to a clinical pharmacologist. ${ }^{3}$ We report here the five year follow up of 41 psychiatric outpatients referred by their general practitioners who took part in a controlled withdrawal programme from long term treatment with diazepam in $1980-2 .^{+}$

\section{Patients, methods, and results}

The 41 patients ( 15 men, 26 women, mean age 43.6 years) had been diagnosed as having generalised anxiety (30), agoraphobia (7), depressive neurosis (2), social phobia (1), or obsessive compulsive neurosis (1) and had taken diazepam (mean $11.3 \mathrm{mg} /$ day) for a mean of 37.5 months before gradual reduction with placebo substitution. In the original study 36 had completed withdrawal and five failed to stop their drugs completely. Follow up was carried out by $\mathrm{AH}$ without knowledge of their original state. Whenever possible patients were interviewed exactly five years after attempted withdrawal and their drug history checked by examining general practice, medical, and psychiatric records (when relevant).

Two of the 41 patients had died in the follow up period. Thirty eight of the remaining 39 patients were seen by $\mathrm{AH}$ and full prescribing details were obtained on all patients (table). Thirty one of the 41 patients had taken benzodiazepines at some time in the five years, although only 14 were still taking them at follow up. Four other patients took other drugs (dothiepin, propranolol, trifluoperazine, and dichloralphenazone) for anxiety symptoms at some time without taking benzodiazepines. There were 73 episodes of new prescribing of benzodiazepines, 69 from general practitioners, comprising diazepam (34), lorazepam (11), chlordiazepoxide (8), temazepam (8), clobazam (5), nitrazepam (3), oxazepam (2), and medazepam (2), all in doses at or below $15 \mathrm{mg}$ of diazepam equivalents per day. In 13 of these episodes the drug was taken for insomnia only, in the remainder for anxiety and stress symptoms.

\section{Psychotropic drug treatment of 41 patients in five year follow up period}

Outcome No of patients

No psychotropic drugs at any time

Other psychotropic drugs but no benzodiazepines

Initial failure to stop diazepam but stopped during follow up

Took benzodiazepines for some time ( 2 weeks-1 year) $)^{\star}$ but stopped before end of five years

Restarted benzodiazepines and was taking them at follow up

^ Distributed between year 1 ( 3 patients), year 2(7), year 3(3), year 4(1), and year $5(2)$

\section{Comment}

It is disturbing that, despite the widespread publicity about benzodiazepine dependence during the period of follow up, most of the patients received a prescription for a benzodiazepine. None the less, the practice of short term prescription was successful in many cases and not followed by a second period of dependence, and no patients required specialist help in coming off their drugs after their participation in the initial study. The continued use of benzodiazepines may also reflect the absence of satisfactory alternatives; once patients have derived benefit from, or been dependent on, benzodiazepines other drug and psychological treatments are rarely deemed a satisfactory substitute at times of subsequent stress. Although the patients' opinions were not sought formally at interview, none regarded the represcription of a benzodiazepine as wrong, although several were not aware of the nature of the drugs they had received.

The results suggest that benzodiazepines may still have a place in treating patients who have been taking long term treatment in the past. Although their use in 